\title{
Color Holographic Reconstruction Using Multiple SLMs and LED Illumination
}

\author{
Fahri Yaraş, Levent Onural \\ Department of Electrical and Electronics Engineering, Bilkent University
}

\begin{abstract}
A color holographic reconstruction technique by using three light emitting diodes (LEDs) is described. Reflective type phase-only spatial light modulators (SLMs) are used since they are suitable for in-line phase holograms. Gerchberg-Saxton iterative algorithm is used for computing phase holograms. Three phase holograms are calculated separately for red, green and blue colors, for a color reconstruction, and separately loaded to corresponding SLMs. Three LEDs are used for illuminating those phase holograms and reconstructions are combined and captured. Experimental results are satisfactory.
\end{abstract}

Keywords: holographic displays, phase holograms, three-dimensional displays, spatial light modulators, computer generated holography

\section{INTRODUCTION}

Phase-only spatial light modulators (SLMs) are promising devices for holographic displays, and phase-only in-line holograms have many desirable features. Some advantages of phase holograms can be listed as high-diffraction efficiency, low-power diffraction orders and low-power undiffracted beam. ${ }^{1}$ Usually lasers are used in various electro-holographic applications; as a consequence of coherence, speckle noise corrupts the reconstructions. In addition to this adverse effect, there is also retina damage risk associated with coherent light. ${ }^{2}$ An alternative is to use light emitting diodes (LEDs) for reconstructions; this will eliminate the speckle, and safe for direct visualization. However, reduced coherence properties might result in loss of reconstructed image quality. Furthermore, compared to lasers, LEDs are readily available, low cost and easy to operate. In Ref.3 monochromatic holographic reconstruction by a light emitting diode is described. They presented a simple setup for color electroholography using three color LEDs. Further developments are shown in Ref.4. In Ref.5-7 other color holographic systems are described.

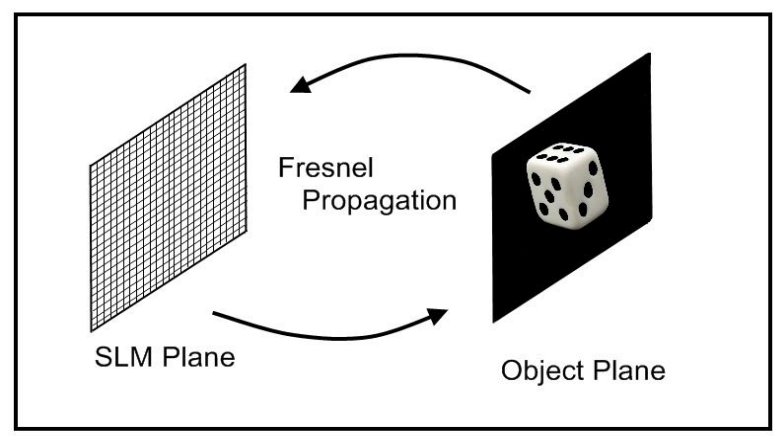

Figure 1. SLM and Object planes

Further author information:

F.Y.: fahri@ee.bilkent.edu.tr

L.O.: onural@bilkent.edu.tr

This work is supported by EC within FP7 under Grant 216105 with the acronym Real 3D.

Stereoscopic Displays and Applications XX, edited by Andrew J. Woods, Nicolas S. Holliman,

John O. Merritt, Proceedings of SPIE-IS\&T Electronic Imaging, SPIE Vol. 7237, 723700

(C) 2009 SPIE-IS\&T · CCC code: 0277-786X/09/\$18 - doi: 10.1117/12.806875 


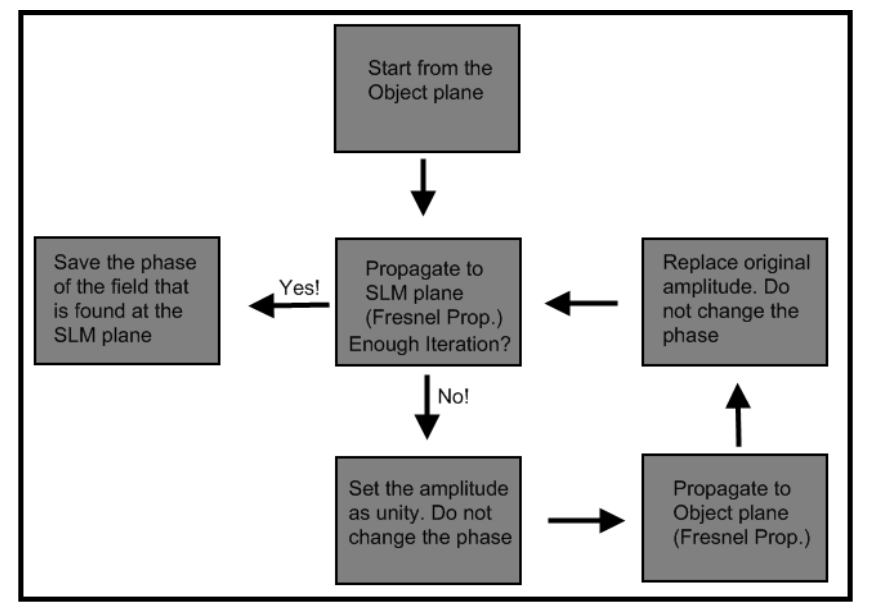

Figure 2. Block diagram of Gerchberg-Saxton algorithm for computing phase holograms

Here in this paper we report experimental color holographic reconstruction results using multiple SLMs and LED illumination. In our experiments iterative algorithm using Fresnel propagation ${ }^{8-11}$ was used to calculate in-line phase holograms. We used three phase-only SLMs and three different color LEDs to have full color holographic reconstructions. The computer graphic card drives the SLMs and red, green and blue channels are controlled in parallel. We used three separate but synchronized SLMs for red, green and blue channels. First, an in-line phase hologram for each channel was computed. Then we combined each phase hologram into an RGB image and output the resultant color hologram pattern via graphic card to the SLMs. After the three phase holograms are loaded to the SLMs, each was illuminated by the corresponding LED. Precisely aligned optical reconstructions from each hologram constitute the color reconstruction which was captured by a CCD array without using any supporting optics.

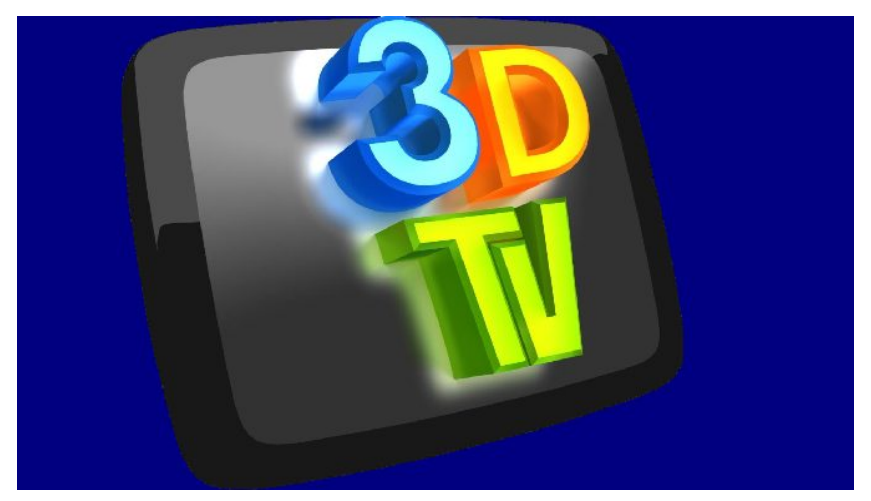

Figure 3. 2D picture of 3DTV logo

\section{EXPERIMENTAL SETUP AND ALGORITHMS}

In our experiments we used phase holograms that are generated using Gerchberg-Saxton iterative algorithm. ${ }^{9,11}$ As shown in Fig. 1 there are two planes. The SLM plane hosts the calculated phase hologram. The object plane represents a 2D amplitude-only object. We used Fresnel approximation ${ }^{8,10}$ for computationally propagating light back and forth between the SLM plane and the object plane. As seen in Fig.2 we started from object plane and propagated the desired amplitude distribution to the SLM plane. At the SLM plane, we do not change the phase but impose constant (unity) amplitude constraint. This simulates a uniform plane wave illumination of the SLM during the optical reconstructions. Then we propagated the complex field at the SLM plane to the 
object plane again. At this stage we impose another amplitude constraint: the computed amplitude is discarded by substituting it with the given object amplitude. Then the new complex field is propagated back to the SLM plane. The iterations continue until the desired object amplitude is obtained. It is important to note that we never alter the phase at any stage of the algorithm. When iterations are over, the phase of the resultant complex field at the SLM plane is saved. For color phase holograms, first we separate the color image into red, blue and green components. Then we apply the algorithm separately for each component. Resultant phase holograms are combined into an RGB image. With the help of the computer graphic card, holograms that are calculated for different wavelengths are easily directed to the corresponding SLMs.

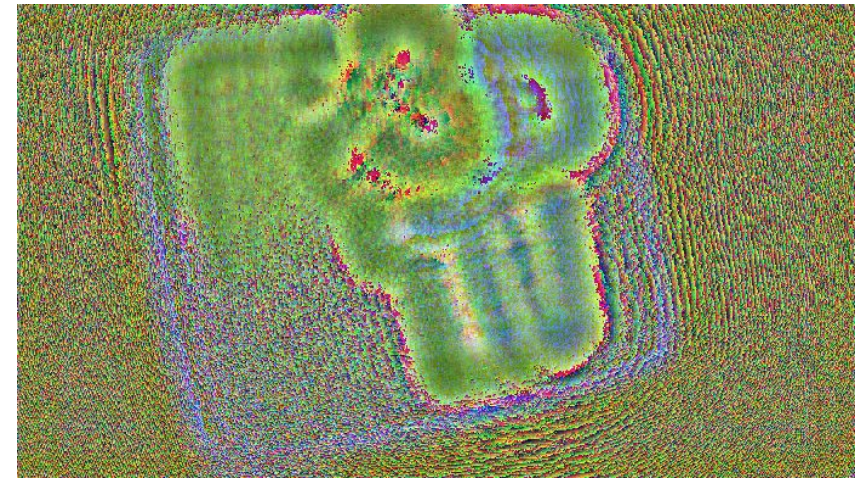

Figure 4. Color phase hologram, containing red, green and blue channel information

As seen in Fig.7 we have used three 1W Edixeon LEDs as light sources. The red, blue and green wavelengths are around $625 \mathrm{~nm}, 460 \mathrm{~nm}$ and $520 \mathrm{~nm}$, respectively. The emitted light from LEDs are passed through a pinhole to narrow the spectrum further and the beam expanders are used for uniform planar illumination. After setting up all LEDs, pinholes, expanders and SLMs, reconstructed color components are combined by using beam splitters. With the help of high precision stages, we adjusted and overlapped all three colors to have an optical colored reconstruction.

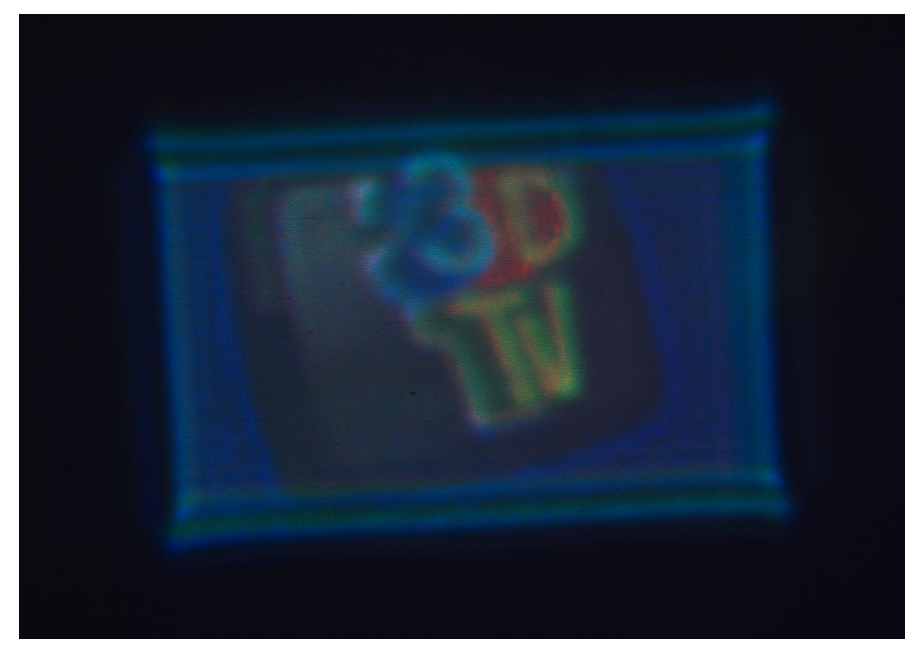

Figure 5. Optical reconstruction from phase hologram

\section{RESULTS}

In our experiments we used HoloEye's HEO1080P phase-only spatial light modulators. Number of pixels is 1920x 1080 with $8 \mu m \times 8 \mu m$ square pixel area. Reconstructed images were taken with a 11 megapixel CCD array 
without using any supporting optics. The reconstructed image size is in the order of the SLM size (about $1.5 \mathrm{x}$ $1.0 \mathrm{~cm}$ ) and the distance of the reconstructed image from the SLM is about $60 \mathrm{~cm}$. In Fig. 3 the original 2D object is shown. Fig. 5 and Fig. 6 show optical and computer reconstructions from the color phase hologram shown in Fig.4, respectively. It is a simple $2 \mathrm{D}$ object and the phase hologram was calculated by 250 iterations.

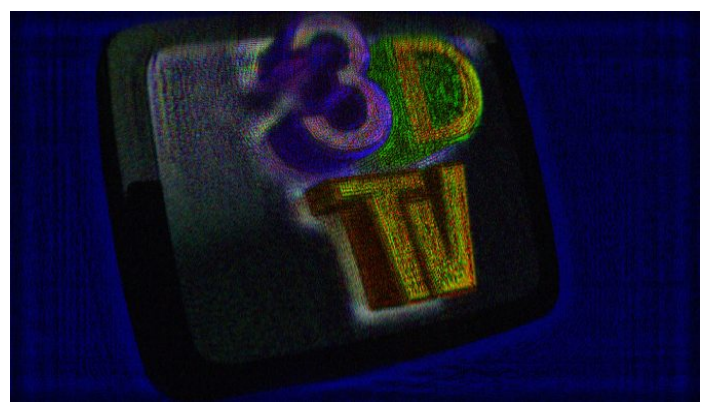

Figure 6. Computer reconstruction from phase hologram

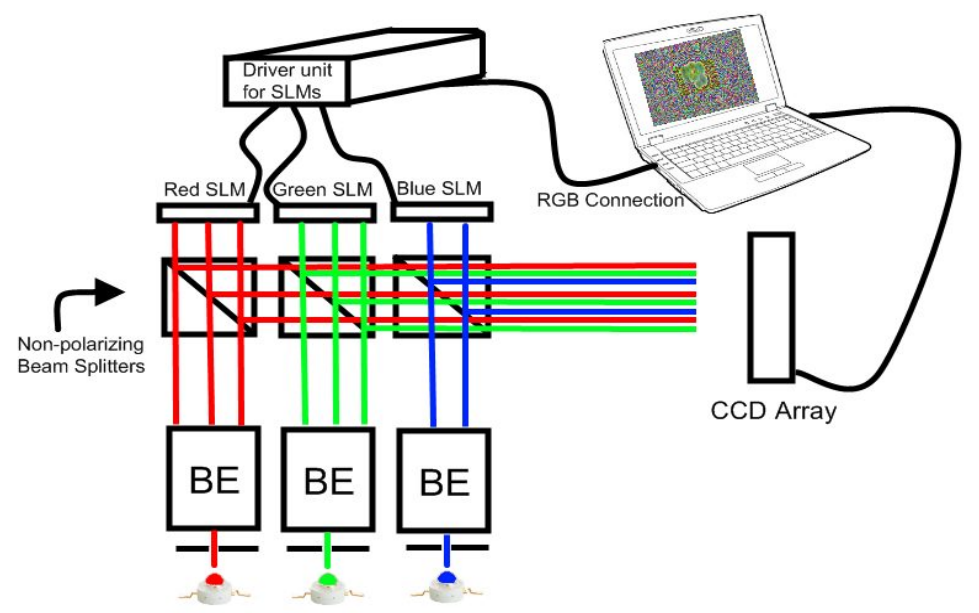

Figure 7. Optical setup. BE: Beam Expander

\section{CONCLUSIONS}

It is observed that the properties of LEDs are sufficient for holographic reconstructions from phase-only SLMs. The quality of color holographic reconstructions, using three phase-only SLMs where each SLM is illuminated by a different color (red, green and blue) LED, is satisfactory.

Though computationally demanding, iterative algorithms like the Gerchberg-Saxton algorithm can be used to compute phase-only computer generated holograms.

Optical alignment is crucial for color holographic reconstruction. In order to simplify this process, test objects like square or several dots can be used.

The presented layout can be used as a color holographic video display. 


\section{REFERENCES}

[1] Lesem, L., Hirsch, P., and Jr., J. J., "The kinoform: a new wave front reconstruction device," IBM J. Res. Dev. 13, 150155 (1969).

[2] Barkana, Y. and Belkin, M., "Laser eye injuries," Survey of Ophthalmology 44(6), 459 - 478 (2000).

[3] Ito, T., Shimobaba, T., Godo, H., and Horiuchi, M., "Holographic reconstruction with a $10-\mu m$ pixel-pitch reflective liquid-crystal display by use of a light-emitting diode reference light," Opt. Letters 27, 1406-1408 (2002).

[4] Ito, T. and Okano, K., "Color electroholography by three colored reference lights simultaneously incident upon one hologram panel," Opt. Express 12, 4320-4325 (2004).

[5] Shimobaba, T., Shiraki, A., Masuda, N., and Ito, T., "An electroholographic colour reconstruction by time division switching of reference lights," J. Opt. A: Pure Appl. Opt. 9, 757-760 (2007).

[6] Yamaguchi, T., Okabe, G., and Yoshikawa, H., "Real-time image plane full-color and full-parallax holographic video display system," Opt. Engineering 46, 125801 (2007).

[7] Makowski, M., Sypek, M., and Kolodziejczyk, A., "Colorful reconstructions from a thin multi-plane phase hologram," Opt. Express 16, 11618-11623 (2008).

[8] Goodman, J., [Introduction to Fourier Optics], McGraw-Hill, New York (1968).

[9] Liu, G. and Scott, P. D., "Phase retrieval and twin-image elimination for in-line fresnel holograms," J. Opt. Soc. Am. 4, 159 (1987).

[10] Onural, L. and Ozaktas, H., "Signal processing issues in diffraction and holographic 3dtv," Signal Processing: Image Communication 22(2), 169-177 (2007).

[11] Gerchberg, R. W. and Saxton, W. O., "A practical algorithm for the determination of the phase from image and diffraction plane pictures," Optik 35, 706-713 (1972). 